Celal Bayar University Journal of Science

\title{
Numerical Solutions of System of First Order Normalized Linear Differential Equations by Using Bernoulli Matrix Method
}

\author{
Kübra ERDEM BİÇER ${ }^{1(D)}$, Mehmet SEZER ${ }^{1 *(D)}$ Mustafa KAZAZ1 $^{\text {(iD) }}$ \\ ${ }^{1}$ Manisa Celal Bayar University Faculty of Art and Science, Manisa, Turkey \\ *kubra.erdem@cbu.edu.tr \\ *Orcid: 0000-0002-4998-6531 \\ Received: 23 December 2020 \\ Accepted: 21May 2021 \\ DOI: $10.18466 /$ cbayarfbe. 845017
}

\begin{abstract}
Systems of first order differential equations have been arisen in science and engineering. Specially, the systems of normalized linear differential equations appear in differential geometry and kinematics problems. Since it is difficult to find solutions to these equations analytically, numerical methods are needed for the approximate solutions. In this study, we find the approximate solutions of the Frenet-Like system with variable coefficients upon the initial conditions by means of a matrix method related to the truncated Bernoulli series. This method transforms the mentioned problem into a system of algebraic equations by using the matrix relations and collocation points; so, the required results along with the solutions are obtained and the usability of the method is discussed.
\end{abstract}

Keywords: Approximate Solutions, Bernoulli polynomials and series, Curves of constant breadth, Matrix Methods, Systems of first order differential equations.

\section{Introduction}

The systems of differential equations in the normal form usually appear in the concept of differential geometry. For instance, a system of differential equations characterizing $E^{4}$ spherical curves can be given as

$$
\frac{d \rho}{d s}=\tau f, \frac{d f}{d s}=-\tau \rho+\mu g, \frac{d g}{d s}=-\mu f,
$$

where $s$ is arc parameter, $\rho(s)=1 / \kappa(s)$ is curvature diameter; $\kappa, \tau$ and $\mu$ are curvatures and, $f(s)$ and $g(s)$ are in the class of $C^{2}[1,2]$.

The system charactering curves of constant breadth are the same type and can be given as

$$
\frac{d \lambda}{d \theta}=\mu, \frac{d \mu}{d \theta}=-\lambda+\rho \tau \delta, \frac{d \delta}{d \theta}=-\rho \tau \mu,
$$

where $\theta(s)=\int_{0}^{s} \kappa(s) d s$ and $\lambda(\theta), \mu(\theta)$ and $\delta(\theta)$ are the coefficients of the curve [3,4].

Besides, the well known Serret-Frenet Equations

$$
\frac{d \vec{t}}{d s}=\kappa \vec{n}, \frac{d \vec{n}}{d s}=-\kappa \vec{t}+\kappa_{2} \vec{b}, \frac{d \vec{b}}{d s}=-\kappa_{2} \vec{n},
$$

lead us to the system of differential equations $[5,6]$ :

$$
\frac{d \varphi_{1}}{d s}=\kappa \varphi_{2}, \frac{d \varphi_{2}}{d s}=-\kappa \varphi_{1}+\tau \varphi_{3}, \frac{d \varphi_{3}}{d s}=-\tau \varphi_{2},
$$

where $\kappa_{1}$ and $\kappa_{2}$ are Euclidean curvatures. The solution of this system also gives as a criterian for periodicity of a space curve. Since the normal systems (1), (2) and (3) same type, it is possible to form them as

$$
\left.\begin{array}{l}
\frac{d y_{1}}{d x}=a(x) y_{2}(x) \\
\frac{d y_{2}}{d x}=-a(x) y_{1}(x)+b(x) y_{3}(x) \\
\frac{d y_{3}}{d x}=-b(x) y_{2}(x)
\end{array}\right\} .
$$

Also the normal system is obtained as

$$
\left.\begin{array}{l}
\frac{d T}{d s}=k_{1} N_{1}+k_{2} N_{2} \\
\frac{d N_{1}}{d s}=-k_{1} T \\
\frac{d N_{2}}{d s}=-k_{2} T
\end{array}\right\},
$$

where 
$k_{1}=\kappa \cos (\theta) \quad, \quad k_{2}=\kappa \sin (\theta) \quad$ and $\quad T=T$, $N_{1}=N \cos \theta-B \sin \theta, \quad N_{2}=N \sin \theta-B \cos \theta$ for the Bishop frame. It can be shown that

$$
\begin{gathered}
\kappa(s)=\sqrt{k_{1}^{2}+k_{2}^{2}}, \theta(s)=\arctan \left(\frac{k_{2}}{k_{1}}\right) \text { and } \\
\tau(s)=-\frac{d \theta(s)}{s} \text { [7]. }
\end{gathered}
$$

These type systems given in (4) and (5) which are called Frenet-Like system is a class of linear differential equations in normal form

$$
\left.\begin{array}{l}
\frac{d y_{1}}{d s}=P_{11}(s) y_{1}(s)+P_{12}(s) y_{2}(s)+P_{13}(s) y_{3}(s)+g_{1}(s) \\
\frac{d y_{2}}{d s}=P_{21}(s) y_{1}(s)+P_{22}(s) y_{2}(s)+P_{23}(s) y_{3}(s)+g_{2}(s) \\
\frac{d y_{3}}{d s}=P_{31}(s) y_{1}(s)+P_{32}(s) y_{2}(s)+P_{33}(s) y_{3}(s)+g_{3}(s)
\end{array}\right\},
$$

or briefly

$$
\frac{d y_{j}}{d s}=\sum_{k=1}^{3} P_{j k}(s)+y_{k}(s)+g_{j}(s) ; \quad j=1,2,3
$$

where $P_{k j}(s)$ and $g(s)$ are functions on interval $0 \leq a \leq s \leq b$.

In this study, we have developed a method based on Bernoulli polynomials to solve system of linear differential equations. Also this method has been used to solve high-order linear differential-difference equations, linear delay difference equations with variable coefficients and mixed linear Fredholm integrodifferential-difference equations, hyperbolic partial differential equations, Helmholtz equations and general functional integro-differential equations with hybrid delays [8-13]. Also many numerical methods have been developed to solve similar equation models [14-20].

We firstly consider the system of differential equations in normal form (6) with the initial conditions

$$
y_{1}(a)=\lambda_{1}, y_{2}(a)=\lambda_{2}, y_{3}(a)=\lambda_{3} ; 0 \leq a \leq s \leq b .
$$

A matrix method is developed to find the approximate solution set in the truncated Bernoulli series form

$$
y_{j}(s)=\sum_{n=0}^{N} a_{j n} B_{n}(s) ; 0 \leq a \leq s \leq b,
$$

where $a_{j n}, j=1,2,3$ are unknown Bernoulli coefficients, $\lambda_{j}, j=1,2,3$ are the given real constants and $B_{n}(s), n=0,1, \ldots, N$. Bernoulli polynomials are defined by [21]

$$
\frac{t e^{x t}}{e^{t}-1}=\sum_{n=0}^{\infty} \frac{B_{n}(x)}{n !} t^{n}
$$

or

$$
B_{n}(x)=\sum_{r=0}^{n}\left(\begin{array}{l}
n \\
r
\end{array}\right) b_{r} x^{n-r} ; \quad b_{r}=B_{r}(0)
$$

Also, an explicit formula for the Bernoulli polynomials is given by

$$
B_{n}^{\prime}(s)=n B_{n-1}(s),
$$

where $B_{0}(s)=1, B_{0}^{\prime}(s)=1, B_{1}(s)=s-\frac{1}{2}, B_{1}^{\prime}(s)=1$

\section{Fundamental matrix relations and Bernoulli matrix method}

In this section, a new matrix technique is devoloped by considering Taylor and Bernoulli collocation methods [22-23] to obtain the approximate solution of the system (6) under the condition (7). For this aim, we compose the matrix form of (6), (7) and (8); firstly the matrix form of (6) can be expressed as

$$
Y^{\prime}(s)=P(s) Y(s)+G(s) ; 0 \leq a \leq s \leq b,
$$

where

$$
\begin{gathered}
Y^{\prime}(s)=\left[\begin{array}{l}
y_{1}^{\prime}(s) \\
y_{2}^{\prime}(s) \\
y_{3}^{\prime}(s)
\end{array}\right], P(s)=\left[\begin{array}{lll}
P_{11}(s) & P_{12}(s) & P_{13}(s) \\
P_{21}(s) & P_{22}(s) & P_{23}(s) \\
P_{31}(s) & P_{32}(s) & P_{33}(s)
\end{array}\right], \\
Y(s)=\left[\begin{array}{l}
y_{1}(s) \\
y_{2}(s) \\
y_{3}(s)
\end{array}\right], G(s)=\left[\begin{array}{l}
g_{1}(s) \\
g_{2}(s) \\
g_{3}(s)
\end{array}\right] .
\end{gathered}
$$

Then the matrix form of (8) can be written as

$$
y_{j}(s)=B(s) A_{j},(j=1,2,3),
$$

where

$$
\begin{gathered}
B(s)=\left[\begin{array}{llll}
B_{0}(s) & B_{1}(s) & \ldots & B_{N}(s)
\end{array}\right], \\
A_{j}=\left[\begin{array}{llll}
a_{j 0} a_{j 1} & a_{j 2} & \ldots & a_{j N}(s)
\end{array}\right]^{T} .
\end{gathered}
$$

Also by using the expressions (10) and (12), we have the matrix form

$$
\begin{aligned}
y_{j}^{\prime}(s) & =B^{\prime}(s) A_{j} \\
& =B(s) D A_{j} ; \quad(j=1,2,3),
\end{aligned}
$$

where

$$
\boldsymbol{D}=\left[\begin{array}{ccccc}
0 & 1 & 0 & \ldots & 0 \\
0 & 0 & 2 & \ldots & 0 \\
\vdots & \vdots & \vdots & \ddots & \vdots \\
0 & 0 & 0 & 0 & \mathrm{~N} \\
0 & 0 & 0 & 0 & 0
\end{array}\right]
$$

By using the matrix relations (12) and (13) into the matrices $Y(s)$ and $Y^{\prime}(s)$, the following matrix forms are obtained as 


$$
\begin{gathered}
Y(s)=\left[\begin{array}{l}
y_{1}(s) \\
y_{2}(s) \\
y_{3}(s)
\end{array}\right]=\left[\begin{array}{l}
B(s) A_{1} \\
B(s) A_{2} \\
B(s) A_{3}
\end{array}\right]=\bar{B}(s) A, \\
Y^{\prime}(s)=\left[\begin{array}{l}
y_{1}^{\prime}(s) \\
y_{2}^{\prime}(s) \\
y_{3}{ }^{\prime}(s)
\end{array}\right]=\left[\begin{array}{l}
B(s) D A_{1} \\
B(s) D A_{2} \\
B(s) D A_{3}
\end{array}\right]=\overline{B(s) D A},
\end{gathered}
$$

where

$$
\begin{gathered}
\bar{B}(s)=\left[\begin{array}{ccc}
B(s) & 0 & 0 \\
0 & B(s) & 0 \\
0 & 0 & B(s)
\end{array}\right], \\
\overline{B(s) D}=\left[\begin{array}{ccc}
B(s) D & 0 & 0 \\
0 & B(s) D & 0 \\
0 & 0 & B(s) D
\end{array}\right], \\
A=\left[\begin{array}{c}
A_{1} \\
A_{2} \\
A_{3}
\end{array}\right], A_{j}=\left[\begin{array}{c}
a_{j 0} \\
a_{j 1} \\
\vdots \\
a_{j N}
\end{array}\right], j=1,2,3 .
\end{gathered}
$$

By substituting the collocation points defined by

$$
s_{i}=a+\frac{b-a}{N} i,(i=0,1, \ldots, N),
$$

into Eq. (11) along with (14), we gain the system of matrix equations

$$
Y^{\prime}\left(s_{i}\right)=P\left(s_{i}\right) Y\left(s_{i}\right)+G\left(s_{i}\right),(i=0,1, \ldots, N),
$$

or briefly, the fundamental matrix equation

$$
Y^{\prime}=P Y+G \Rightarrow(\overline{B D}-P \bar{B}) A=G,
$$

where

$$
\begin{gathered}
P=\left[\begin{array}{cccc}
P\left(s_{0}\right) & 0 & \cdots & 0 \\
0 & P\left(s_{0}\right) & \cdots & 0 \\
\vdots & \vdots & \ddots & \vdots \\
0 & 0 & \cdots & P\left(s_{N}\right)
\end{array}\right], G=\left[\begin{array}{c}
G\left(s_{0}\right) \\
G\left(s_{1}\right) \\
\vdots \\
G\left(s_{N}\right)
\end{array}\right], \\
Y=\left[\begin{array}{c}
Y\left(s_{0}\right) \\
Y\left(s_{1}\right) \\
\vdots \\
Y\left(s_{N}\right)
\end{array}\right]=\left[\begin{array}{c}
\bar{B}\left(s_{0}\right) A \\
\bar{B}\left(s_{1}\right) A \\
\vdots \\
\bar{B}\left(s_{N}\right) A
\end{array}\right]=\bar{B} A, \\
Y^{\prime}(s)=\left[\begin{array}{c}
Y^{\prime}\left(s_{0}\right) \\
Y^{\prime}\left(s_{1}\right) \\
\vdots \\
Y^{\prime}\left(s_{N}\right)
\end{array}\right]=\left[\begin{array}{c}
\overline{B\left(s_{0}\right) D} A \\
\overline{B\left(s_{1}\right) D} A \\
\vdots \\
\overline{B\left(s_{N}\right) D} A
\end{array}\right]=\overline{B D} A .
\end{gathered}
$$

In Eq. (13), the full dimensions of the matrices $\overline{B D}, P, \bar{B}, A, \quad$ and $\quad G \quad$ are $3(N+1) \times 3(N+1)$, $3(N+1) \times 3(N+1), 3(N+1) \times 3(N+1), 3(N+1) \times 1 \quad$ and $3(N+1) \times 1$, respectively.
The fundamental matrix equation (15) corresponding to Eq. (4) can be written in the compact form

$$
W A=G \text { or }[W ; G] \text {, }
$$

where

$$
W=\overline{B D}-P \bar{B}=\left[w_{p q}\right] ; p, q=1,2, \ldots, 3(N+1) .
$$

The matrix equation (16) corresponds to a system of a linear algebraic equations in $3(N+1)$ unknown Bernoulli coefficients.

By using the initial conditions defined by (7) and the matrix relation $Y(s)$ in (14), we obtain the matrix form for the conditions as

$$
\bar{B}(a) A=\lambda \Leftrightarrow[\bar{B}(a) ; \lambda],
$$

where

$$
\bar{B}(a)=\left[\begin{array}{ccc}
B(a) & 0 & 0 \\
0 & B(a) & 0 \\
0 & 0 & B(a)
\end{array}\right], \lambda=\left[\begin{array}{l}
\lambda_{1} \\
\lambda_{2} \\
\lambda_{3}
\end{array}\right] .
$$

Consequently, we obtain the new following augmented matrix for the problem (6)-(7) by replacing the row matrices (17) with the rows involving the coefficients $a_{10}, a_{20}$ and $a_{30}$ of the matrix (16):

$$
[\tilde{W} ; \tilde{G}] \text { or } \tilde{W} A=\tilde{G} .
$$

If $\operatorname{rank} \quad \tilde{\mathbf{W}}=\operatorname{rank}[\tilde{\mathbf{W}} ; \tilde{\mathbf{G}}]=\mathrm{N}+1$, the unknown Bernoulli coefficient matrix can be written as $\mathbf{A}=(\tilde{\mathbf{W}})^{-1} \tilde{\mathbf{G}}$ and the approximate solution in the Bernoulli matrix form is obtained as

$$
\begin{gathered}
Y(s)=\bar{B}(s) A, \\
y_{j}(s) \cong \sum_{n=0}^{N} a_{j n} B_{n}(s) .
\end{gathered}
$$

\section{Results and Discussion}

In this section two examples are given to demonstrate the applicability of this method. These examples have been calculated by using Matlab.

Example 1. Consider the system of first-order linear differential equations

$$
\begin{array}{ll}
\frac{d y_{1}}{d x}=x y_{2}(x)-x^{2} & \\
\frac{d y_{2}}{d x}=-x y_{1}(x)+y_{3}(x) & 0 \leq x \leq 1,
\end{array}
$$

$$
\frac{d y_{3}}{d x}=-y_{2}(x)+x+1
$$

with the initial conditions $y_{1}(0)=1, y_{2}(0)=0, y_{3}(0)=1$ which has the exact solution $y_{1}(x)=1, y_{2}(x)=x, y_{3}(x)=x+1$. 
The approximate solution is $y_{j}(s)=\sum_{n=0}^{2} a_{j n} B_{n}(s)$, $j=1,2,3$. The collocation points for $N=2$ are computed as $\left\{x_{1}=0, x_{2}=\frac{1}{2}, x_{3}=1\right\}$.

The fundamental matrix equation is

$$
W A=(\overline{B D}-P \bar{B}) A=G,
$$

where

$$
\overline{B D}=\left[\begin{array}{ccccccccc}
0 & 1 & -1 & 0 & 0 & 0 & 0 & 0 & 0 \\
0 & 0 & 0 & 0 & 1 & -1 & 0 & 0 & 0 \\
0 & 0 & 0 & 0 & 0 & 0 & 0 & 1 & -1 \\
0 & 1 & 0 & 0 & 0 & 0 & 0 & 0 & 0 \\
0 & 0 & 0 & 0 & 1 & 0 & 0 & 0 & 0 \\
0 & 0 & 0 & 0 & 0 & 0 & 0 & 1 & 0 \\
0 & 1 & 1 & 0 & 0 & 0 & 0 & 0 & 0 \\
0 & 0 & 0 & 0 & 1 & 1 & 0 & 0 & 0 \\
0 & 0 & 0 & 0 & 0 & 0 & 0 & 1 & 1
\end{array}\right],
$$$$
P=\left[\begin{array}{ccccccccc}
0 & 0 & 0 & 0 & 0 & 0 & 0 & 0 & 0 \\
0 & 0 & 1 & 0 & 0 & 0 & 0 & 0 & 0 \\
0 & -1 & 0 & 0 & 0 & 0 & 0 & 0 & 0 \\
0 & 0 & 0 & 0 & \frac{1}{2} & 0 & 0 & 0 & 0 \\
0 & 0 & 0 & -\frac{1}{2} & 0 & 1 & 0 & 0 & 0 \\
0 & 0 & 0 & 0 & -1 & 0 & 0 & 0 & 0 \\
0 & 0 & 0 & 0 & 0 & 0 & 0 & 1 & 0 \\
0 & 0 & 0 & 0 & 0 & 0 & -1 & 0 & 1 \\
0 & 0 & 0 & 0 & 0 & 0 & 0 & -1 & 0
\end{array}\right],
$$

$$
\bar{B}=\left[\begin{array}{ccccccccc}
1 & -\frac{1}{2} & \frac{1}{6} & 0 & 0 & 0 & 0 & 0 & 0 \\
0 & 0 & 0 & 1 & -\frac{1}{2} & \frac{1}{6} & 0 & 0 & 0 \\
0 & 0 & 0 & 0 & 0 & 0 & 1 & -\frac{1}{2} & \frac{1}{6} \\
1 & 0 & -\frac{1}{12} & 0 & 0 & 0 & 0 & 0 & 0 \\
0 & 0 & 0 & 1 & 0 & -\frac{1}{12} & 0 & 0 & 0 \\
0 & 0 & 0 & 0 & 0 & 0 & 1 & 0 & -\frac{1}{12} \\
1 & \frac{1}{2} & \frac{1}{6} & 0 & 0 & 0 & 0 & 0 & 0 \\
0 & 0 & 0 & 1 & \frac{1}{2} & \frac{1}{6} & 0 & 0 & 0 \\
0 & 0 & 0 & 0 & 0 & 0 & 1 & \frac{1}{2} & \frac{1}{6}
\end{array}\right] .
$$

The augmented matrix for this fundamental matrix equation is calculated as

$$
[W ; G]=\left[\begin{array}{ccccccccccc}
0 & 1 & -1 & 0 & 0 & 0 & 0 & 0 & 0 & ; & 0 \\
0 & 0 & 0 & 0 & 1 & -1 & -1 & \frac{1}{2} & -\frac{1}{6} & ; & 0 \\
0 & 0 & 0 & 1 & -\frac{1}{2} & \frac{1}{6} & 0 & 1 & -1 & ; & 1 \\
0 & 1 & 0 & -\frac{1}{2} & 0 & \frac{1}{24} & 0 & 0 & 0 & ; & -\frac{1}{4} \\
\frac{1}{2} & 0 & -\frac{1}{24} & 0 & 1 & 0 & -1 & 0 & \frac{1}{12} & ; & 0 \\
0 & 0 & 0 & 1 & 0 & -\frac{1}{12} & 0 & 1 & 0 & ; & \frac{3}{2} \\
0 & 1 & 1 & -1 & -\frac{1}{2} & -\frac{1}{6} & 0 & 0 & 0 & ; & -1 \\
1 & \frac{1}{2} & \frac{1}{6} & 0 & 1 & 1 & -1 & -\frac{1}{2} & -\frac{1}{6} & ; & 0 \\
0 & 0 & 0 & 1 & \frac{1}{2} & \frac{1}{6} & 0 & 1 & 1 & ; & 2
\end{array}\right] .
$$

From equation (17), we obtain the matrix form of the initial conditions

$$
[\bar{B}(0) ; \lambda]=\left[\begin{array}{ccccccccccc}
1 & -\frac{1}{2} & \frac{1}{6} & 0 & 0 & 0 & 0 & 0 & 0 & ; & 1 \\
0 & 0 & 0 & 1 & -\frac{1}{2} & \frac{1}{6} & 0 & 0 & 0 & ; & 0 \\
0 & 0 & 0 & 0 & 0 & 0 & 1 & -\frac{1}{2} & \frac{1}{6} & ; & 1
\end{array}\right] .
$$

From equation (18), the new augmented matrix based on the conditions is calculated as

$$
[\tilde{W} ; \tilde{G}]=\left[\begin{array}{ccccccccccc}
1 & -\frac{1}{2} & \frac{1}{6} & 0 & 0 & 0 & 0 & 0 & 0 & ; & 1 \\
0 & 0 & 0 & 1 & -\frac{1}{2} & \frac{1}{6} & 0 & 0 & 0 & ; & 0 \\
0 & 0 & 0 & 0 & 0 & 0 & 1 & -\frac{1}{2} & \frac{1}{6} & ; & 1 \\
0 & 1 & 0 & -\frac{1}{2} & 0 & \frac{1}{24} & 0 & 0 & 0 & ; & -\frac{1}{4} \\
\frac{1}{2} & 0 & -\frac{1}{24} & 0 & 1 & 0 & -1 & 0 & \frac{1}{12} & ; & 0 \\
0 & 0 & 0 & 1 & 0 & -\frac{1}{12} & 0 & 1 & 0 & ; & \frac{3}{2} \\
0 & 1 & 1 & -1 & -\frac{1}{2} & -\frac{1}{6} & 0 & 0 & 0 & ; & -1 \\
1 & \frac{1}{2} & \frac{1}{6} & 0 & 1 & 1 & -1 & -\frac{1}{2} & -\frac{1}{6} & ; & 0 \\
0 & 0 & 0 & 1 & \frac{1}{2} & \frac{1}{6} & 0 & 1 & 1 & ; & 2
\end{array}\right] .
$$

Solving this system, the unknown Bernoulli coefficient matrix is obtained as

$$
A=\left[\begin{array}{lllllllll}
1 & 0 & 0 & \frac{1}{2} & 1 & 0 & \frac{3}{2} & 1 & 0
\end{array}\right]^{T} .
$$

By substituting the Bernoulli coefficient matrix into equation (12), we obtain the approximate solution set $y_{1}(x)=1, y_{2}(x)=x, y_{3}(x)=x+1$ which is the exact solution. 
Example 2. Consider the curve $\alpha:[0,2] \rightarrow E_{1}^{3}$ given by

$$
\alpha(s)=\left(\frac{s^{3}}{3}+s, \frac{s^{3}}{3}, \frac{s^{2}}{\sqrt{2}}\right) \text {. }
$$

The system of differential equations in the normal form corresponding to curve $\alpha$ is as follows [24]

$$
\begin{aligned}
& \lambda_{1}^{\prime}(s)=-\sqrt{2} \cos (\sqrt{2} s) \lambda_{2}(s)+\sqrt{2} \sin (\sqrt{2} s) \lambda_{3}(s) \\
& \lambda_{2}^{\prime}(s)=-\sqrt{2} \cos (\sqrt{2} s) \lambda_{1}(s) \\
& \lambda_{3}^{\prime}(s)=\sqrt{2} \sin (\sqrt{2} s) \lambda_{1}(s)
\end{aligned}
$$

The approximate solution set of this problem is solved using Bernoulli Collocation method with the initial conditions $\lambda_{1}(0)=1, \lambda_{2}(0)=2$ and $\lambda_{3}(0)=3$. For initial conditions value of distance $d$ is

$$
d=\sqrt{\left|-\lambda_{1}^{2}+\lambda_{2}^{2}+\lambda_{3}^{2}\right|}=\sqrt{12} \cong 3.464101615 \text {. [16] }
$$

Solving the problem (20) in the same way as Section 2 for $N=3$, we get

$$
\begin{aligned}
& \lambda_{1}(s)=11.1592106 s^{3}-15.4680213 s^{2}+ \\
& 18.7854967 s+0.999999995 \\
& \lambda_{2}(s)=10.77765 s^{3}-26.4167678 s^{2}+ \\
& 15.1614944 s+2 \\
& \lambda_{3}(s)=10.1776605 s^{3}-13.7259918 s^{2}+ \\
& 17.6088868 s+2.99999997 .
\end{aligned}
$$

Hence the value of distance $d$ for obtained approximate solution is

$$
d=\sqrt{\left|-\lambda_{1}^{2}+\lambda_{2}^{2}+\lambda_{3}^{2}\right|}=\sqrt{12} \cong 3.464101615 .
$$

Let's briefly summarize the results given in this article as follows:

In section 1 , the problem and Bernoulli polynomials have been introduced. In section 2 , the matrix relations of the given problem has been obtained and Bernoulli matrix method has been developed. In section 3, two examples have been considered to demonstrate accuracy of the method. In section 4 , obtained results have been examined and the advantage of the method have been highlighted.

\section{Conclusion}

In this study, system of Frenet-Like differential equations which arise in differential geometry as a model for linear equation systems are discussed. To solve this equation system, a numerical methos has been developed. This method is based on Bernoulli polynomials and collocations points. To demonstrate the applicability of the present method, two examples have been considered. Obtained results have been showed that the method is suitable for the solutions of differential equation systems. One of the important advantages of this method is that solutions are obtained very easily and practically by using computer programs. Since linear differential equations are encountered in many physics, chemistry, biology and engineering problems, this method can be extended another models with small modifications, which is another advantage of the method.

\section{Author's Contributions}

Kübra Erdem Biçer: Made literature search, performed numerical examples.

Mehmet Sezer: Supervised the manuscript and drafted the manuscript.

Mustafa Kazaz: Helped in results interpretation, performed the analysis.

\section{Ethics}

There are no ethical issues after the publication of this manuscript.

\section{References}

1. Dannon, V. 1981. Integral Characterizations and Theory of Curves. Proc. Amer. Math. Soc.; 4: 600-603.

2. Sezer, M. 1989. Differential Equations and Integral Characterizations for E4- Spherical Curves. Doga Tr. J. Math.; 13: $125-131$

3. Köse, Ö. 1986. On Space Curves of Constant Breadth. Doga Tr. J. Math.; 10: 11-14.

4. Sezer, M. 1989. Differential Equations Characterizing Space Curves of Constant Breadth and A Criterion for These Curves. Doga Tr. J. Math.; 13: 70-78.

5. Do Carmo, MP. Differential Geometry of Curves and Surfaces. Prentice Hall, Inc. Englewood Cliffs, 1976.

6. Paşalı Atmaca, S, Akgüller, Ö, Sezer, M. 2013. Integral Characterization of a System of Differential Equations and Applications. Nonl. Analysis and Differential Equations; 1(2): $57-$ 66

7. Cetin, M, Tunçer, Y, Karacan, MK. 2014. Smarandache Curves According to Bishop Frame in Euclidean 3-Space. Gen. Math. Notes; 20(2): 50-66.

8. Erdem, K, Yalçinbaş, S. 2012. Numerical approach of linear delay difference equations with variable coefficients in terms of Bernoulli polynomials. AIP Conf. Proc.; 1493: 338-344.

9. Erdem, K, Yalçinbaş, S. 2012. Bernoulli Polynomial Approach to High-Order Linear Differential Difference Equations. AIP Conf. Proc.; 1479: 360-364.

10. Erdem, K, Yalçinbaş, S, Sezer, M. 2013. A Bernoulli approach with residual correction for solving mixed linear Fredholm integro-differential-difference equation. Journal of Difference Equations and Applications; 19(10): 1619-1631.

11. Erdem, K, Yalçinbaș, S. 2016. A matrix approach to solving hyperbolic partial differential equations using Bernoulli polynomials. Filomat; 30(4): 993-1000 
12. Erdem, K, Yalçinbaş, S. 2017. Numerical Solutions for Helmholtz Equations using Bernoulli Polynomials. AIP Conf. Proc.; 1863: 300021-1-300021-4.

13. Erdem Biçer, K, Sezer, M. 2017. Bernoulli Matrix-Collocation Method for solving General Functional Integro-Differential Equations with Hybrid Delays. Journal of Inequalities and Special Functions; 8(3): 85-99.

14. Ahmad H, Khan, TA, Durur, H, İsmail, GM, Yokus, A. 2021. Analytic approximate solutions of diffusion equations arising in oil pollution. Journal of Ocean Engineering and Science; 6(1): 62-69.

15. Rasool, G, Zhang, T, Shafiq, A, Durur, H. 2019. Influence of chemical reaction on Marangoni convective flow of nanoliquid in the presence of Lorentz forces and thermal radiation: A numerical investigation. Journal of Advances in Nanotechnology; 1(1): 32.

16. Ahmad, H, Rafiq, M, Cesarano, C, Durur, H. 2020. Variational iteration algorithm-I with an auxiliary parameter for solving boundary value problems. Earthline Journal of Mathematical Sciences; 3(2): 229-247.

17. Yavuz, M, Yokus, A. 2020. Analytical and numerical approaches to nerve impulse model of fractional-order. Numerical Methods for Partial Differential Equations; 36(6): 1348-1368.

18. Durur, H, Senol, M, Kurt, A, Tasbozan, O. 2019. Approximate solutions of the time fractional Kadomtsev-Petviashvili equation with conformable derivative. Erzincan Univ. J. Sci. Technol; 12(2): 796-806.

19. Yokus, A. 2020. Truncation and convergence dynamics: KdV burgers model in the sense of Caputo derivative. Boletim da Sociedade Paranaense de Matematica; doi: 10.5269/bspm.47472.

20. Rasool, G, Shafiq, A, Durur, H. 2020. Darcy-Forchheimer relation in Magnetohydrodynamic Jeffrey nanofluid flow over stretching surface. Discrete \& Continuous Dynamical Systems-S doi: $10.3934 /$ dcdss.2020399.

21. Apostol, TM. Introduction to Analytic Number Theory, SpringerVerlag, New York, 1976, pp 264-267.

22. Ates, BY, Çetin, M, Sezer, M. 2015. Taylor polynomial approach for systems of linear differential equations in normal form and residual error estimation. NTMSCI; 3: 116-128.

23. Sahiner, B, Sezer, M. 2018. Determining constant breadth curve mate of a curve on a surface via Taylor collocation method. Determining constant breadth curve mate of a curve on a surface via Taylor collocation method. NTMSCI; 6(3): 103-115.

24. Cetin, M. Sabit Genişlikli Eğriler Ve Küresel Eğrilerin Diferensiyel Karakterizasyonlar. PhD Thesis, Manisa Celal Bayar University, The Institute of Natural and Applied Sciences, 2015. 